Corrigendum

\title{
Corrigendum to "New Mechanisms of Tumor-Associated Macrophages on Promoting Tumor Progression: Recent Research Advances and Potential Targets for Tumor Immunotherapy"
}

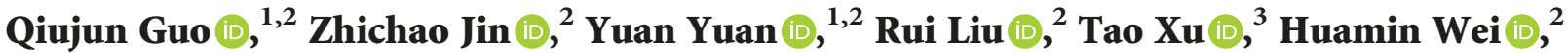 \\ Xinyao Xu $\mathbb{D}^{1,2}$ Shulin He, ${ }^{1,2}$ Shuntai Chen $\mathbb{D}^{1,2}{ }^{1,2}$ Zhan Shi $\mathbb{D}^{4}{ }^{4}$ Wei Hou, ${ }^{2}$ and Baojin Hua ${ }^{2}$ \\ ${ }^{1}$ Beijing University of Chinese Medicine, No. 11 North Third Ring Road East, Chaoyang District, Beijing 100029, China \\ ${ }^{2}$ Department of Oncology, Guang'anmen Hospital, China Academy of Chinese Medicine Sciences, No. 5 Beixiange, Xicheng District, \\ Beijing 100053, China \\ ${ }^{3}$ Department of Oncology, Xiyuan Hospital, China Academy of Chinese Medicine Sciences, No. 1 Playground Road, Haidian District, \\ Beijing 100091, China \\ ${ }^{4}$ Institute of Basic Research in Clinical Medicine (IBRCM), China Academy of Chinese Medicine Sciences, No. 16 Dongzhimen \\ Nanxiaojie, Dongcheng District, Beijing 100700, China \\ Correspondence should be addressed to Qiujun Guo; drguoqiujun@126.com
}

Received 4 June 2018; Accepted 2 July 2018; Published 29 July 2018

Copyright (c) 2018 Qiujun Guo et al. This is an open access article distributed under the Creative Commons Attribution License, which permits unrestricted use, distribution, and reproduction in any medium, provided the original work is properly cited.

In the article titled "New Mechanisms of Tumor-Associated Macrophages on Promoting Tumor Progression: Recent Research Advances and Potential Targets for Tumor Immunotherapy" [1], the order of the first two affiliations was reversed. The revised authors' list and affiliations are shown above.

\section{References}

[1] Q. Guo, Z. Jin, Y. Yuan et al., "New mechanisms of tumorassociated macrophages on promoting tumor progression: recent research advances and potential targets for tumor immunotherapy," Journal of Immunology Research, vol. 2016, Article ID 9720912, 12 pages, 2016. 


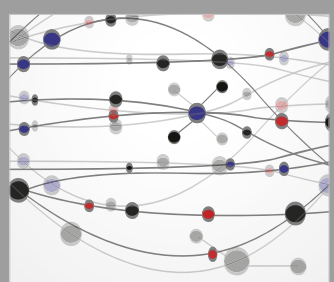

The Scientific World Journal
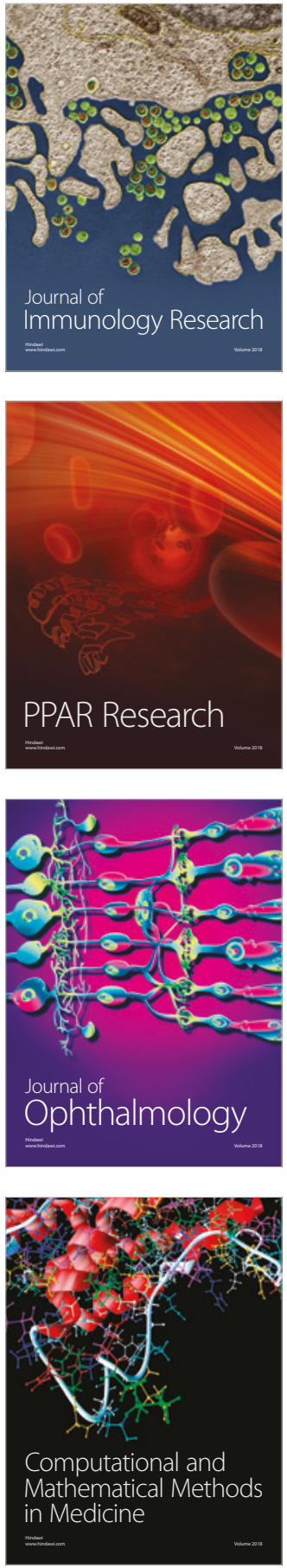

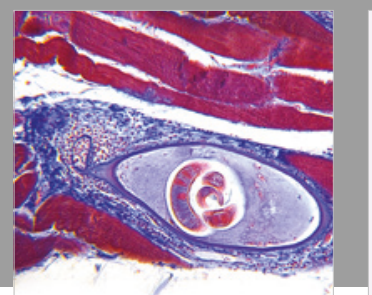

Gastroenterology Research and Practice

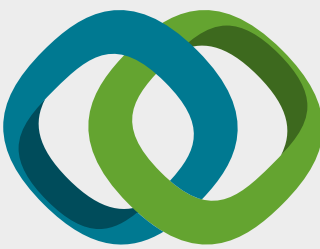

\section{Hindawi}

Submit your manuscripts at

www.hindawi.com
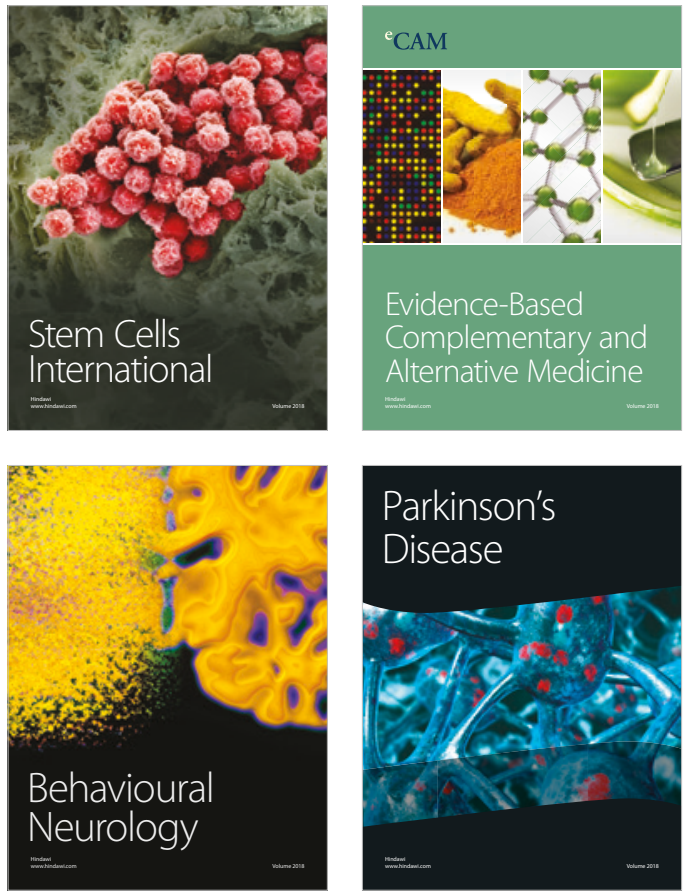

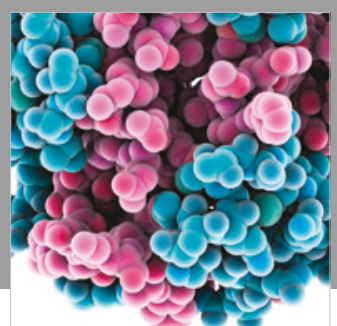

ournal of

Diabetes Research

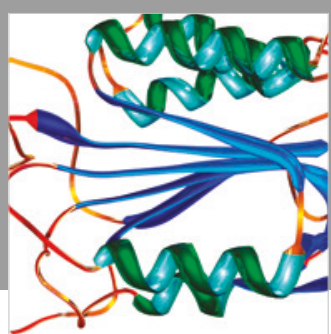

Disease Markers
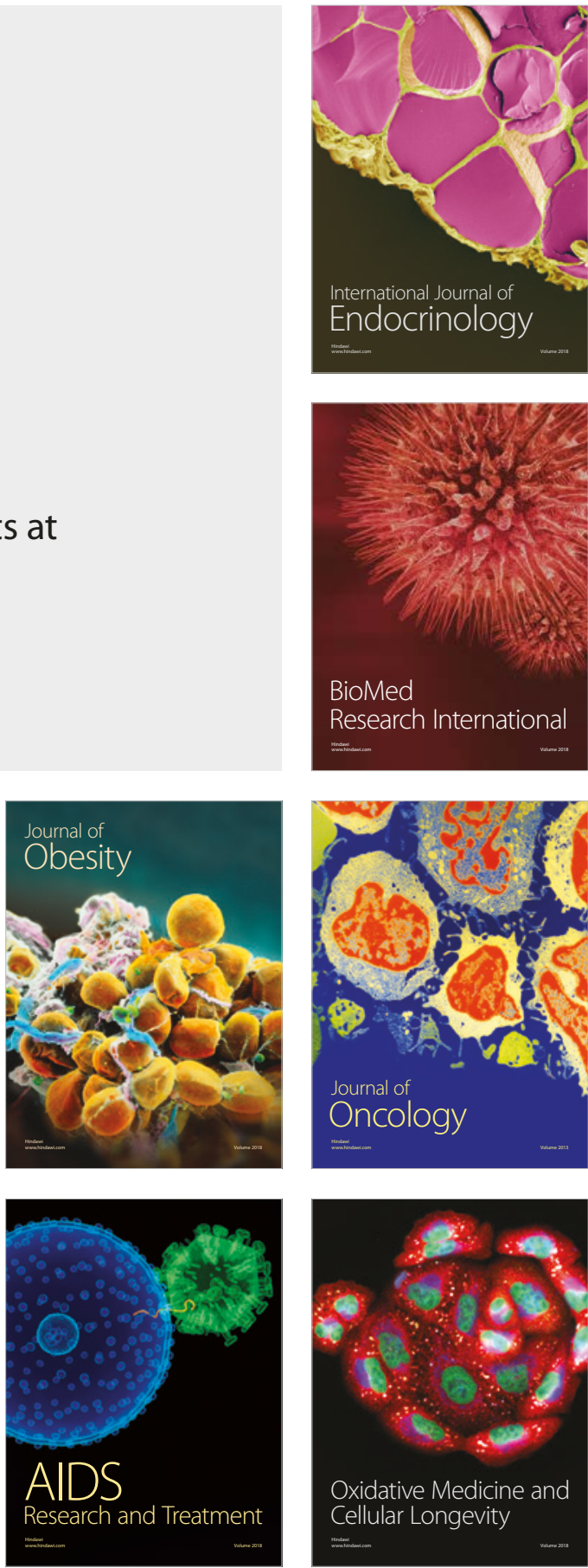